TRANSACTIONS OF THE

AMERICAN MATHEMATICAL SOCIETY

Volume 356, Number 6, Pages 2149-2168

S 0002-9947(03)03395-6

Article electronically published on December 9, 2003

\title{
HARDY INEQUALITIES WITH OPTIMAL CONSTANTS AND REMAINDER TERMS
}

\author{
FILIPPO GAZZOLA, HANS-CHRISTOPH GRUNAU, AND ENZO MITIDIERI
}

\begin{abstract}
We show that the classical Hardy inequalities with optimal constants in the Sobolev spaces $W_{0}^{1, p}$ and in higher-order Sobolev spaces on a bounded domain $\Omega \subset \mathbb{R}^{n}$ can be refined by adding remainder terms which involve $L^{p}$ norms. In the higher-order case further $L^{p}$ norms with lower-order singular weights arise. The case $1<p<2$ being more involved requires a different technique and is developed only in the space $W_{0}^{1, p}$.
\end{abstract}

\section{INTRODUCTION}

Let $n>2$ and let $\Omega \subset \mathbb{R}^{n}$ be a bounded domain. A well-known Hardy inequality (see $[\mathrm{H}],[\mathrm{HLP}]$ ), which can be considered one of the really classical Sobolev embedding inequalities, reads as:

$$
\int_{\Omega}|\nabla u|^{2} d x \geq \frac{(n-2)^{2}}{4} \int_{\Omega} \frac{u^{2}}{|x|^{2}} d x \quad \text { for all } u \in W_{0}^{1,2}(\Omega) .
$$

Although it is not explicitly assumed that $0 \in \Omega$, here and in what follows we shall always have this particularly interesting case in mind. Related $L^{p}$-versions of (1) are also well known, and the simplest can be stated as follows:

$$
\int_{\Omega}|\nabla u|^{p} d x \geq\left(\frac{n-p}{p}\right)^{p} \int_{\Omega} \frac{|u|^{p}}{|x|^{p}} d x \quad \text { for all } u \in W_{0}^{1, p}(\Omega) .
$$

Here, we are assuming that $n>p \geq 1$. A first higher-order generalization of (1) was proved by Rellich in $[\mathbb{R}$ :

$$
\int_{\Omega}(\Delta u)^{2} d x \geq \frac{n^{2}(n-4)^{2}}{16} \int_{\Omega} \frac{u^{2}}{|x|^{4}} d x \quad \text { for all } u \in W_{0}^{2,2}(\Omega),
$$

where $n>4$. Recently some extensions of (3) appeared in [DH], [Mi]. These results allow us to estimate integral terms of the form $\int_{\Omega}|x|^{-\sigma}(\Delta u)^{2} d x$ and consequently to prove Hardy inequalities in higher-order Sobolev spaces $W_{0}^{k, 2}(\Omega)$, where $n>2 k$.

If $k=2 m$ is even, we have

$$
\int_{\Omega}\left(\Delta^{m} u\right)^{2} d x \geq\left(\prod_{\ell=1}^{2 m} \frac{(n+4 m-4 \ell)^{2}}{4}\right) \int_{\Omega} \frac{u^{2}}{|x|^{4 m}} d x,
$$

Received by the editors June 20, 2000 and, in revised form, May 8, 2003.

2000 Mathematics Subject Classification. Primary 46E35; Secondary 35B50, 35J40.

(C)2003 American Mathematical Society 
while if $k=2 m+1$ is odd,

$$
\int_{\Omega}\left|\nabla \Delta^{m} u\right|^{2} d x \geq\left(\prod_{\ell=1}^{2 m+1} \frac{(n+4 m+2-4 \ell)^{2}}{4}\right) \int_{\Omega} \frac{u^{2}}{|x|^{4 m+2}} d x .
$$

In the $L^{p}$-setting further types of Rellich inequalities were also proved in [DH] p. 520], [Mi]. A simple example in this direction is given by

$$
\int_{\Omega}|\Delta u|^{p} d x \geq\left(\frac{(n-2 p)(p-1) n}{p^{2}}\right)^{p} \int_{\Omega} \frac{|u|^{p}}{|x|^{2 p}} d x \quad \text { for all } u \in W_{0}^{2, p}(\Omega),
$$

where $n>2 p$. We point out that all the constants appearing in (3), (4), (5) and (6) are sharp. For a more extensive survey, bibliography and historical remarks we refer again to $[\mathrm{DH}]$.

Refined versions of Hardy inequalities seem to have first appeared in Ma, Section 2.1.6, Corollary 3]. This kind of inequalities was applied by Brezis and Vazquez BV] to the Gelfand problem

$$
-\Delta u=\lambda \exp (u), \quad u \geq 0, \quad u \in W_{0}^{1,2}(\Omega),
$$

where $\lambda$ is a positive parameter and $n>2$. It is well known that there exists $\lambda^{*}>0$ such that for $0 \leq \lambda \leq \lambda^{*}$ equation (7) is solvable, while for $\lambda>\lambda^{*}$ it has no solutions. Further, for $\lambda=2(n-2)$ and $\Omega=B$ the unit ball, problem (7) has the following explicit singular solution:

$$
u_{\text {sing }}=-2 \log |x| \in W_{0}^{1,2}(B) .
$$

The linearization of equation (7) around $u_{\text {sing }}$ leads to the "Hardy-type" operator

$$
L_{\operatorname{lin}} \varphi=-\Delta \varphi-\frac{2(n-2)}{|x|^{2}} \varphi \text {. }
$$

This operator is studied in $[\mathrm{BV}]$ for proving, among other results, in which dimensions the singular solution is also extremal in the sense that it corresponds to $\lambda=\lambda^{*}$. This is shown to be precisely the case if $L_{\text {lin }}$ is positive semidefinite. In order to decide whether the latter holds true, Brezis and Vazquez used the following Hardy inequality with optimal constant and a remainder term:

$$
\int_{\Omega}|\nabla u|^{2} d x \geq \frac{(n-2)^{2}}{4} \int_{\Omega} \frac{u^{2}}{|x|^{2}} d x+\Lambda_{2}\left(\frac{e_{n}}{|\Omega|}\right)^{2 / n} \int_{\Omega} u^{2} d x \quad \text { for all } u \in W_{0}^{1,2}(\Omega) .
$$

Here $n \geq 2$. $\Lambda_{2}$ denotes the first eigenvalue of the Laplace operator in the twodimensional unit disk and $e_{n},|\Omega|$ denote respectively the $n$-dimensional Lebesgue measure of the unit ball $B \subset \mathbb{R}^{n}$ and of the domain $\Omega$. Inequality (8) can be proved by means of a "reduction of the dimension" argument. This technique was implicitly used also in [Ma, Section 2.1.6]. For further applications and variants of (8) we refer to $\mathrm{VZ}$.

Remainder terms appear also in other Sobolev inequalities and in the context of nonlinear eigenvalue problems; see e.g. [BL, GG].

The goal of this paper is to prove the existence of remainder terms for all Hardytype inequalities (2), (3), (4), (5) and (6) mentioned above.

We feel that remainder terms in the Rellich inequality (3) may find an interpretation for the biharmonic analogue of the Gelfand problem;

$$
\Delta^{2} u=\lambda \exp (u), \quad u \geq 0, \quad u \in W_{0}^{2,2}(\Omega) .
$$


In AGGM], we started the investigation of (9) when $\Omega$ is a ball, since in this case comparison principles for the biharmonic operator under Dirichlet boundary conditions are available. This problem is rather involved, and up to now, we did not succeed in making the connection between (9) and the improved Hardy inequalities explicit.

In Section 2 we shall study $W_{0}^{1, p}$-Hardy type inequalities corresponding to the $p$ Laplace operator $\Delta_{p}$. Carefully exploiting the convexity properties of the function $\xi \mapsto \xi^{p}$, we show that an extra $L^{p}$-norm may be added to the right hand side of (2). Up to purely $p$-dependent factors, the constant in front of this remainder term behaves like $n^{(p-2)}$ as $n \rightarrow \infty$, and it vanishes when $p \searrow 1$.

Although the problem of finding optimal constants for the remainder terms in the inequalities is very interesting, our choice is not to develop this kind of study in this paper. However, we believe that the asymptotic behaviour of such constants as $n \rightarrow \infty$ is precisely as stated above.

Closely related questions were studied simultaneously and independently by Adimurthi, Chaudhuri and Ramaswamy [ACR].

In Sections 3 and 4 we show that remainder terms of the form

$$
\int_{\Omega} u^{2} d x, \ldots, \int_{\Omega} \frac{u^{2}}{|x|^{2 k-2}} d x
$$

can be added to the right hand sides of (4) and (5). In Section 3 we shall consider functions satisfying Navier boundary conditions, that is $0=u=\Delta u=\cdots$ on $\partial \Omega$, while in Section 4 we deal with functions satisfying Dirichlet boundary conditions $0=u=\nabla u=\Delta u=\cdots$ on $\partial \Omega$. In Section 3 we also present a refinement of the Rellich inequality (6) in the $L^{p}$-setting for $p \geq 2$. For $1<p<2$ we expect a similar phenomenon as in $W_{0}^{1, p}$, concerning the behaviour of the constants appearing in the remainder terms. See Theorem 2 and the subsequent remark for more details on this point.

Since the class of functions considered in Section 3 is larger than the class considered in Section 4, the constants in front of the remainder terms are smaller than those in Section 4 Moreover, the constants which appear in the latter seem to be more natural when the order $k$ of the Sobolev space becomes large. Next we observe that the proofs of the results in Section 3 are easily reduced to the "spherically symmetric case", while it appears that this reduction when dealing with functions satisfying Dirichlet boundary conditions is more involved. This is due to the fact that the symmetrization argument used in Section 3 fails, and the iterative procedures we use, seem to produce only relatively small constants. For this reason it may be also of interest in its own to study how the result in $W_{0}^{k, 2}(B)$ for radial functions can be extended to nonsymmetric functions.

\section{An $L^{p}$-REMAINDER TERM FOR THE HARDY INEQUALITY IN $W_{0}^{1, p}(\Omega)$}

In this section we shall prove that a remainder term can be added to the right hand side of (2). In what follows we shall always suppose that $1<p<n$.

We set

$$
X:=\left\{v \in C^{1}([0,1]): v^{\prime}(0)=v(1)=0\right\} \backslash\{0\},
$$

and define the constants

$$
\lambda_{p}=\inf _{X} \frac{\int_{0}^{1} r^{p-1}\left|v^{\prime}(r)\right|^{p} d r}{\int_{0}^{1} r^{p-1}|v(r)|^{p} d r}, \quad \Lambda_{p}=\inf _{X} \frac{\int_{0}^{1} r|v(r)|^{p-2}\left(v^{\prime}(r)\right)^{2} d r}{\int_{0}^{1} r^{p-1}|v(r)|^{p} d r}
$$


and

$$
\Gamma=\Gamma(n, p)=\max \left\{\lambda_{p},(p-1)\left(\frac{n-p}{p}\right)^{p-2} \Lambda_{p}\right\} .
$$

Remark. For all $p>1$ we have $\lambda_{p}>0$, and if $p=2$, then $\Gamma=\Lambda_{2}=\lambda_{2}$. Moreover, if $p \in \mathbb{N}$ then $\lambda_{p}$ is the first eigenvalue of $-\Delta_{p}$ in the unit ball contained in $\mathbb{R}^{p}$. In addition, a simple integration by parts argument and an application of Hölder's inequality show that $\lambda_{p}, \Lambda_{p} \geq 1$. Therefore, $\Gamma \geq c_{p} n^{p-2}$ as $n \rightarrow \infty$.

Theorem 1. Let $\Omega \subset \mathbb{R}^{n}$ be a bounded domain and $p \in(1, n)$. Let $e_{n}=\left|B_{1}(0)\right|$ and let $|\Omega|$ be the $n$-dimensional Lebesgue measure of the unit ball and of the domain $\Omega$ respectively.

(a) If $p \geq 2$, then for every $u \in W_{0}^{1, p}(\Omega)$ we have

$$
\int_{\Omega}|\nabla u|^{p} d x \geq\left(\frac{n-p}{p}\right)^{p} \int_{\Omega} \frac{|u|^{p}}{|x|^{p}} d x+\Gamma \cdot\left(\frac{e_{n}}{|\Omega|}\right)^{p / n} \int_{\Omega}|u|^{p} d x .
$$

(b) If $1<p<2$, then there exists a constant $C=C(n, p)>0$ such that for every $u \in W_{0}^{1, p}(\Omega)$ the following inequality holds:

$$
\int_{\Omega}|\nabla u|^{p} d x \geq\left(\frac{n-p}{p}\right)^{p} \int_{\Omega} \frac{|u|^{p}}{|x|^{p}} d x+C \cdot\left(\frac{e_{n}}{|\Omega|}\right)^{p / n} \int_{\Omega}|u|^{p} d x .
$$

Remarks. (i) The explicit form of the constant $C(n, p)$ appearing in (11) is given in (19) below. We point out that the asymptotic behaviour of this constant as $n \rightarrow \infty$ is given, up to an $n$-independent factor, by $n^{p-2}$. Although we do not claim that the constants $\Gamma(n, p)$ and $C(n, p)$ are sharp, we believe that they reflect the precise asymptotic behaviour for $n \rightarrow \infty$.

(ii) Following the generalizations in $[\mathrm{BV}]$ and $[\mathrm{VZ}]$ we expect that also the $p$-th power of any $W^{1, q}$-norm with $q<p$ or of the $L^{r}$-norm of $u$ with $r<\frac{n p}{n-p}$ may serve as a remainder term in (10) and (11). In this case the constants appearing in front of these remainder terms will be not so simple looking even for $p \geq 2$. After the present paper was submitted for publication, some results in this direction were obtained in $\mathrm{ACR}$, [BFT]. For improved Hardy inequalities involving a whole series of remainder terms see [FT].

(iii) If $p=1$ and $\Omega=B_{1}(0)$ the Hardy constant

$$
n-1=\inf _{u \in W_{0}^{1,1}\left(B_{1}(0)\right) \backslash\{0\}} \frac{\int|\nabla u| d x}{\int \frac{|u|}{|x|} d x}
$$

is achieved by any positive smooth and radially decreasing function $u$ with $\left.u\right|_{|x|=1}=0$. This means that for $p=1$ no remainder term may be added to the corresponding Hardy inequality and shows that necessarily $C(n, p) \searrow 0$ as $p \searrow 1$.

(iv) For related inequalities with weights being the inverse of the distance function $\delta$, i.e. $\delta(x)=d(x, \partial \Omega)$, where $\Omega$ is a bounded domain contained in $\mathbb{R}^{n}$, we refer to $[\overline{\mathrm{BM}}],[\mathrm{BMS}],[\mathrm{MS}]$. A unified approach to this kind of inequalities has been given recently in $[\mathrm{BFT}]$, where the distance function from any $k$-codimensional manifold, $1 \leq k \leq n$, is considered. 
To prove Theorem 1 we need the following elementary inequalities, which give a quantitative estimate from below for the convexity behaviour of power-like functions. Similar inequalities in a more general context but with smaller constants were obtained in $[\mathrm{L}]$ and several subsequent papers.

Lemma 1. Let $p \geq 1$ and $\xi, \eta$ be real numbers such that $\xi \geq 0$ and $\xi-\eta \geq 0$. Then

$$
(\xi-\eta)^{p}+p \xi^{p-1} \eta-\xi^{p} \geq \begin{cases}\max \left\{(p-1) \eta^{2} \xi^{p-2},|\eta|^{p}\right\}, & \text { if } p \geq 2, \\ \frac{1}{2} p(p-1) \frac{\eta^{2}}{(\xi+|\eta|)^{2-p}}, & \text { if } 1 \leq p \leq 2 .\end{cases}
$$

The proof can be obtained as an application of Taylor's formula. The interested reader may refer to the appendix of this paper for further details.

Proof of Theorem 1. Using Schwarz symmetrization and a rescaling argument, we may assume that $\Omega$ is the unit ball $B=B_{1}(0)$, and that $u \in W_{0}^{1, p}(\Omega)$ is nonnegative, radially symmetric and nonincreasing. We recall that symmetrization leaves $L^{q}$-norms of functions invariant, it increases $L^{p}$-norms with the singular weight $|x|^{-p}$ (see e.g. [AL, Theorem 2.2]), and decreases $L^{p}$-norms of the gradient (see [AL, Theorem 2.7]). By density, we may further assume that $u$ is as smooth as needed.

So, in what follows we may write $u(r), u^{\prime}(r)=\frac{d}{d r} u(r)$. Clearly, we have $|\nabla u(x)|=\left|u^{\prime}(r)\right|$ with $r=|x|$. Our goal is to find a lower bound for

$$
I:=\int_{0}^{1} r^{n-1}\left|u^{\prime}(r)\right|^{p} d r-\left(\frac{n-p}{p}\right)^{p} \int_{0}^{1} r^{n-p-1} u(r)^{p} d r .
$$

Similarly as in BV] a suitable transformation "reduces" the dimension and also uncovers a remainder term. We set

$$
v(r):=r^{(n / p)-1} u(r), \quad u(r)=r^{1-(n / p)} v(r),
$$

so that

$$
u^{\prime}(r)=-\frac{n-p}{p} r^{-n / p} v(r)+r^{1-(n / p)} v^{\prime}(r) .
$$

Since $u$ is radially nonincreasing, for $r \in(0,1]$ we have

$$
\frac{n-p}{p} \cdot \frac{v(r)}{r} \geq v^{\prime}(r)
$$

and $I$ becomes

$$
I=\int_{0}^{1} r^{p-1}\left(\frac{n-p}{p} \cdot \frac{v(r)}{r}-v^{\prime}(r)\right)^{p} d r-\left(\frac{n-p}{p}\right)^{p} \int_{0}^{1} \frac{v(r)^{p}}{r} d r .
$$

Since (13) holds, we can apply Lemma 1 with the choice

$$
\xi=\frac{n-p}{p} \cdot \frac{v(r)}{r} \quad \text { and } \quad \eta=v^{\prime}(r) .
$$


We first consider $p \geq 2$. Using the boundary condition $v(0)=v(1)=0$, we obtain

$$
\begin{aligned}
I \geq & -p\left(\frac{n-p}{p}\right)^{p-1} \int_{0}^{1} v(r)^{p-1} \cdot v^{\prime}(r) d r \\
& +\max \left\{(p-1)\left(\frac{n-p}{p}\right)^{p-2} \int_{0}^{1} r v(r)^{p-2}\left(v^{\prime}(r)\right)^{2} d r, \int_{0}^{1} r^{p-1}\left|v^{\prime}(r)\right|^{p} d r\right\} \\
\geq & \max \left\{(p-1)\left(\frac{n-p}{p}\right)^{p-2} \Lambda_{p}, \lambda_{p}\right\} \int_{0}^{1} r^{p-1} v(r)^{p} d r \\
= & \Gamma \int_{0}^{1} r^{n-1} u(r)^{p} d r
\end{aligned}
$$

and (10) follows.

The case $1<p<2$ requires greater effort. By Lemma 1 and $\int_{0}^{1} v^{p-1} \cdot v^{\prime} d r=0$, it follows that

$$
I \geq \frac{1}{2} p(p-1) \int_{0}^{1} \frac{r^{p-1}\left|v^{\prime}(r)\right|^{2}}{\left(\frac{n-p}{p} \cdot \frac{v(r)}{r}+\left|v^{\prime}(r)\right|\right)^{2-p}} d r
$$

In order to estimate the right hand side of (14) from below we introduce a further regularizing factor $r^{\varepsilon}$. Here for the moment we just require that $\varepsilon>0$. We will see below that a good choice for the value of $\varepsilon$ is given by $\varepsilon=2-p$. As a consequence we will miss $\lambda_{p}$ as a coefficient for the remainder term. Application of Hölder's inequality yields

$$
\begin{aligned}
& \left(\int_{0}^{1} r^{\varepsilon+p-1}\left|v^{\prime}(r)\right|^{p} d r\right)^{2 / p} \\
& =\left(\int_{0}^{1} \frac{r^{p(p-1) / 2}\left|v^{\prime}(r)\right|^{p}}{\left(\frac{n-p}{p} \cdot \frac{v(r)}{r}+\left|v^{\prime}(r)\right|\right)^{(2-p) p / 2}}\right. \\
& \left.\quad \times\left(r^{\varepsilon+(p-1)(2-p) / 2}\left(\frac{n-p}{p} \cdot \frac{v(r)}{r}+\left|v^{\prime}(r)\right|\right)^{(2-p) p / 2}\right) d r\right)^{\frac{2}{p}} \\
& \leq\left(\int_{0}^{1} \frac{r^{p-1}\left|v^{\prime}(r)\right|^{2}}{\left(\frac{n-p}{p} \cdot \frac{v(r)}{r}+\left|v^{\prime}(r)\right|\right)^{2-p}} d r\right) \\
& \quad \times\left(\int_{0}^{1} r^{2 \varepsilon /(2-p)} r^{p-1}\left(\frac{n-p}{p} \cdot \frac{v(r)}{r}+\left|v^{\prime}(r)\right|\right)^{p} d r\right)^{\frac{2-p}{p}} .
\end{aligned}
$$


Proceeding further in estimating the second integral of (15) by means of a Hardy inequality in "dimension $(p+\varepsilon)$ ", we find

$$
\begin{aligned}
& \int_{0}^{1} r^{2 \varepsilon /(2-p)} r^{p-1}\left(\frac{n-p}{p} \cdot \frac{v(r)}{r}+\left|v^{\prime}(r)\right|\right)^{p} d r \\
& \quad \leq 2^{p-1}\left(\frac{n-p}{p}\right)^{p} \int_{0}^{1} r^{\varepsilon+p-1}\left(\frac{v(r)}{r}\right)^{p} d r+2^{p-1} \int_{0}^{1} r^{\varepsilon+p-1}\left|v^{\prime}(r)\right|^{p} d r \\
& \quad \leq 2^{p-1}\left(\left(\frac{n-p}{\varepsilon}\right)^{p}+1\right) \int_{0}^{1} r^{\varepsilon+p-1}\left|v^{\prime}(r)\right|^{p} d r .
\end{aligned}
$$

Here we have used the inequality $(a+b)^{p} \leq 2^{p-1}\left(a^{p}+b^{p}\right)(\forall a, b \geq 0)$ and the fact that $r^{2 \varepsilon /(2-p)}<r^{\varepsilon}$ for all $r \in(0,1)$. Combining the above estimate with (15), inserting the result into (14), and choosing

$$
\varepsilon:=2-p
$$

we arrive at

$$
I \geq \frac{1}{2} p(p-1) 2^{(p-1)(p-2) / p}\left(\left(\frac{n-p}{2-p}\right)^{p}+1\right)^{(p-2) / p} \int_{0}^{1} r\left|v^{\prime}(r)\right|^{p} d r .
$$

On the other hand, by the two-dimensional embedding $W_{0}^{1, p} \subset L^{2 p /(2-p)} \subset L^{p(3-p)}$, we may define the constant

$$
C_{0}(p):=\inf _{X} \frac{\int_{0}^{1} r\left|v^{\prime}(r)\right|^{p} d r}{\left(\int_{0}^{1} r|v(r)|^{p(3-p)} d r\right)^{1 /(3-p)}},
$$

and thus we obtain

$$
\begin{aligned}
\int_{0}^{1} r^{n-1} u(r)^{p} d r & =\int_{0}^{1} r^{p-1} v(r)^{p} d r=\int_{0}^{1} r^{-(p-2)^{2} /(3-p)}\left(r^{1 /(3-p)} v(r)^{p}\right) d r \\
& \leq\left(\int_{0}^{1} r^{p-2} d r\right)^{(2-p) /(3-p)}\left(\int_{0}^{1} r v(r)^{p(3-p)} d r\right)^{1 /(3-p)} \\
& \leq\left(\frac{1}{p-1}\right)^{(2-p) /(3-p)} C_{0}(p)^{-1} \int_{0}^{1} r\left|v^{\prime}(r)\right|^{p} d r .
\end{aligned}
$$

Inserting this estimate into (17), we finally deduce

$$
\int_{B}|\nabla u|^{p} d x \geq\left(\frac{n-p}{p}\right)^{p} \int_{B} \frac{|u|^{p}}{|x|^{p}} d x+C(n, p) \int_{B}|u|^{p} d x
$$

with

$$
C(n, p)=\frac{1}{2} p(p-1)^{1+(2-p) /(3-p)} 2^{(p-1)(p-2) / p}\left(\left(\frac{n-p}{2-p}\right)^{p}+1\right)^{(p-2) / p} C_{0}(p) .
$$

The proof of (11) is now complete.

Remark. Applying the a priori estimates for the $p$-Laplace operator of [To] and [E] to the Euler-Lagrange equations corresponding to (18) it follows that $C_{0}(p) \rightarrow \Lambda_{2}$ as $p \nearrow 2$. Then, by (19), we deduce $C(n, p) \rightarrow \Lambda_{2}=\Gamma(n, 2)$ as $p \nearrow 2$. Moreover, $C_{0}(p)$ is uniformly bounded from below for $p \in(1,2]$. Indeed, a rough estimate of $C_{0}(p)$ is given by $C_{0}(p) \geq(2 \pi)^{-1 / 8} \approx 0.8$. 


\section{WEAKLY SINGULAR $L^{p}$-REMAINDER TERMS IN RELLICH-TYPE INEQUALITIES} IN HIGHER-ORDER SOBOLEV SPACES $W^{k, p}(p \geq 2)$

UNDER NAVIER BOUNDARY CONDITIONS

In this section by modifying the "reduction of the dimension" technique used in BV], we shall prove higher-order Rellich-type inequalities in $W^{k, p}$ with remainder terms for functions satisfying Navier boundary conditions.

Let $p \geq 2$. Let $v=v(r)$ be a smooth radially symmetric function and denote by $\Delta_{\vartheta}$ the operator defined by

$$
\Delta_{\vartheta} v:=v^{\prime \prime}+\frac{\vartheta-1}{r} v^{\prime}, \quad \text { where } \vartheta=\vartheta(n, p)=4+\frac{n(p-2)}{p} .
$$

Throughout this section we shall set

$$
X=\left\{v \in C^{2}([0,1]): v^{\prime}(0)=v(1)=0, v \not \equiv 0\right\}
$$

and

$$
\begin{gathered}
\gamma=\inf _{X} \frac{\int_{0}^{1} r\left|v^{\prime}(r)\right|^{2} d r}{\int_{0}^{1} r v(r)^{2} d r}, \quad \Gamma=\max \left\{\frac{(p-1)^{p-1}(n-2 p)^{p-2} n^{p-2}}{p^{2(p-2)}} \Lambda_{\vartheta}, \lambda_{\vartheta}\right\}, \\
\lambda_{\vartheta}=\inf _{X} \frac{\int_{0}^{1} r^{2 p-1}\left|\Delta_{\vartheta} v(r)\right|^{p} d r}{\int_{0}^{1} r^{2 p-1}|v(r)|^{p} d r}, \quad \Lambda_{\vartheta}=\inf _{X} \frac{\int_{0}^{1} r^{3}|v(r)|^{p-2}\left(\Delta_{\vartheta} v(r)\right)^{2} d r}{\int_{0}^{1} r^{2 p-1}|v(r)|^{p} d r} .
\end{gathered}
$$

These notations should not be confused with those of Section 2

Remarks. The value of $\gamma$ does not depend neither on $p$ nor on $n$. Indeed, $\gamma$ is nothing else than the first eigenvalue of $-\Delta$ under homogeneous Dirichlet boundary conditions in the unit disk $B \subset \mathbb{R}^{2} ; \gamma=j_{0}^{2} \approx 2.4^{2}$. Here $j_{\nu}$ denotes the first positive zero of the Bessel function $J_{\nu}$. If $p=2$, then $\Lambda_{\vartheta}=\lambda_{\vartheta}$ and the first eigenvalue of the biharmonic operator under Navier boundary conditions in the unit ball in $\mathbb{R}^{4}$ is given by $\Lambda_{\vartheta}=\lambda_{\vartheta}=j_{1}^{4} \approx 3.8^{4} \approx 215$. With the help of a tedious calculation, which is outlined in Lemma 4 in the appendix, we find positive lower bounds for $\Lambda_{\vartheta}$ and $\lambda_{\vartheta}$ also in the case $p>2$. These bounds imply that if $p>2$, then $\Gamma \geq c_{p} n^{2 p-2}$ for all $n$.

In the space $W^{2, p} \cap W_{0}^{1, p}$ we have the following statement.

Theorem 2. Let $p \geq 2$ and let $\Omega \subset \mathbb{R}^{n}(n \geq 2 p)$ be a bounded domain. Denote by $|\Omega|$ its $n$-dimensional Lebesgue measure and let $e_{n}=|B|$, where $B=B_{1}(0)$ is the unit ball.

Then, for any $u \in W^{2, p} \cap W_{0}^{1, p}(\Omega)$ we have

$$
\begin{aligned}
\int_{\Omega}|\Delta u|^{p} d x \geq & \left(\frac{(n-2 p)(p-1) n}{p^{2}}\right)^{p} \int_{\Omega} \frac{|u|^{p}}{|x|^{2 p}} d x \\
& +\frac{4(p-1)^{p}(n-2 p)^{p-1} n^{p-1}}{p^{2 p-1}} \gamma\left(\frac{e_{n}}{|\Omega|}\right)^{2 / n} \int_{\Omega} \frac{|u|^{p}}{|x|^{2 p-2}} d x \\
& +\Gamma\left(\frac{e_{n}}{|\Omega|}\right)^{2 p / n} \int_{\Omega}|u|^{p} d x
\end{aligned}
$$

Proof.

STEP 1. By scaling it suffices to consider $|\Omega|=e_{n}$. First we show that we may restrict ourselves to $\Omega=B$ and radial superharmonic functions $u$. For this 
purpose we assume that (20) has already been shown in this setting. Now let $\Omega$ be an arbitrary bounded domain with $|\Omega|=e_{n}$ and let $u \in W^{2, p} \cap W_{0}^{1, p}(\Omega)$.

Define $f=-\Delta u$ and let $w \in W^{2, p} \cap W_{0}^{1, p}(B)$ be the radial strong solution of

$$
\begin{cases}-\Delta w=f^{*} & \text { in } B, \\ w=0 & \text { on } \partial B,\end{cases}
$$

where $f^{*} \geq 0$ denotes the Schwarz symmetrization of $f$. By [Ta, Theorem 1] we know that $w \geq u^{*} \geq 0$. Hence

$$
\begin{aligned}
\int_{\Omega}|\Delta u|^{p} d x & =\int_{\Omega}|f|^{p} d x=\int_{B}\left(f^{*}\right)^{p} d x=\int_{B}|\Delta w|^{p} d x, \\
\int_{B} \frac{|w|^{p}}{|x|^{\alpha}} d x & \geq \int_{B} \frac{\left|u^{*}\right|^{p}}{|x|^{\alpha}} d x \geq \int_{\Omega} \frac{|u|^{p}}{|x|^{\alpha}} d x, \quad \alpha \in\{0,2 p-2,2 p\} .
\end{aligned}
$$

For the last inequality we refer again to [AL, Theorem 2.2]. Assuming that (20) holds in the radial superharmonic setting, the same follows for any domain $\Omega$ and any $u \in W^{2, p} \cap W_{0}^{1, p}(\Omega)$.

SteP 2. Assume now that $\Omega=B$ and that $u$ is superharmonic and radially symmetric: $u=u(r), r=|x|$. Let us also recall that in the $W^{2, p}$ setting, the best Hardy constant is given by $C_{p}^{p}$ (see [DH, Theorem 12], [Mi, Theorem 3.1]), where

$$
C_{p}=\frac{(n-2 p)(p-1) n}{p^{2}} .
$$

Similarly as in (12), we introduce the transformation

$$
v(r):=r^{(n / p)-2} u(r), \quad u(r)=r^{2-(n / p)} v(r),
$$

so that

$$
-\Delta u(r)=r^{-n / p}\left(-r^{2} \Delta_{\vartheta} v(r)+C_{p} v(r)\right) \geq 0 .
$$

This allows us to apply Lemma 1 with the choice $\xi=C_{p} v$ and $\eta=r^{2} \Delta_{\vartheta} v$. Indeed, we have

$$
\begin{aligned}
I_{p}:= & \int_{0}^{1} r^{n-1}(-\Delta u)^{p} d r-C_{p}^{p} \int_{0}^{1} r^{n-2 p-1}|u|^{p} d r \\
= & \int_{0}^{1} r^{-1}\left[\left(C_{p} v-r^{2} \Delta_{\vartheta} v\right)^{p}-\left(C_{p} v\right)^{p}\right] d r \\
\geq & -p C_{p}^{p-1} \int_{0}^{1} r v^{p-1} v^{\prime \prime} d r \\
& +\max \left\{(p-1) C_{p}^{p-2} \int_{0}^{1} r^{3}(v(r))^{p-2}\left(\Delta_{\vartheta} v\right)^{2} d r, \int_{0}^{1} r^{2 p-1}\left|\Delta_{\vartheta} v\right|^{p} d r\right\},
\end{aligned}
$$

where we have used the boundary condition $u(1)=v(1)=0$. Integrating by parts and using the identity $v^{p-2}\left|v^{\prime}\right|^{2}=\frac{4}{p^{2}}\left|\left(v^{p / 2}\right)^{\prime}\right|^{2}$ gives

$$
-\int_{0}^{1} r v^{p-1} v^{\prime \prime} d r=(p-1) \int_{0}^{1} r v^{p-2}\left|v^{\prime}\right|^{2} d r=\frac{4(p-1)}{p^{2}} \int_{0}^{1} r\left|\left(v^{p / 2}\right)^{\prime}\right|^{2} d r .
$$


Therefore, recalling the definition of $\gamma, \lambda_{\vartheta}$ and $\Lambda_{\vartheta}$, we infer

$$
\begin{aligned}
I_{p} & \geq \frac{4(p-1)}{p} C_{p}^{p-1} \gamma \int_{0}^{1} r|v|^{p} d r+\max \left\{(p-1) C_{p}^{p-2} \Lambda_{\vartheta}, \lambda_{\vartheta}\right\} \int_{0}^{1} r^{2 p-1}|v|^{p} d r \\
& =\frac{4(p-1)}{p} C_{p}^{p-1} \gamma \int_{0}^{1} r^{n-1} \frac{|u|^{p}}{r^{2 p-2}} d r+\Gamma \int_{0}^{1} r^{n-1}|u|^{p} d r
\end{aligned}
$$

thereby completing the proof of Theorem 2 .

Remark. For the case $1<p<2$, the preceding proof shows the existence of a remainder term $\int_{\Omega} \frac{|u|^{p}}{|x|^{2 p-2}}$ with a factor which depends on $n$ like $(n-2 p)^{(p-1)}$. We are confident that, even in this case, methods similar to those used in the proof of Theorem 1 can be employed to prove that an additional term of the type $\int_{\Omega}|u|^{p}$ may be added to the right hand side of (6).

In order to avoid tedious calculations, from now on we deal only with the Hilbert space case $p=2$. The case $p>2$ is expected to be similar. To make the statements that follow more precise, we introduce some further notations. Set

$$
X=\left\{v \in C^{1}([0,1]): v^{\prime}(0)=v(1)=0, v \not \equiv 0\right\}
$$

and for $n \in \mathbb{N}$ let

$$
\Lambda(n)=\inf _{X} \frac{\int_{0}^{1} r^{n-1}\left|v^{\prime}(r)\right|^{2} d r}{\int_{0}^{1} r^{n-1} v(r)^{2} d r} .
$$

Again, this notation should not be confused with the previous ones.

Proposition 1. Let $B \subset \mathbb{R}^{n}$ be the unit ball. The first eigenvalue $\lambda_{0}$ of the following Navier boundary value problem:

$$
\left\{\begin{array}{l}
\Delta^{2} u=\lambda u \text { in } B \\
u=\Delta u=0 \text { on } \partial B
\end{array}\right.
$$

satisfies

$$
\Lambda(n)^{2}=\lambda_{0}=\inf _{u \in W^{2,2} \cap W_{0}^{1,2}(B) \backslash\{0\}} \frac{\int_{B}(\Delta u)^{2} d x}{\int_{B} u^{2} d x} .
$$

Proof. The first equality is due to the fact that the biharmonic operator $u \mapsto \Delta^{2} u$ under the Navier boundary conditions $u=\Delta u=0$ on $\partial B$ is actually the square of the Laplacian under Dirichlet boundary conditions. For the second equality, see e.g. [V, Lemma B3].

A direct consequence of Theorem 2 is the following corollary.

Corollary 1. Let $\Omega \subset \mathbb{R}^{n}, n \geq 4$, be a bounded domain. Denote by $|\Omega|$ its $n$ dimensional Lebesgue measure and let $e_{n}=|B|$, where $B=B_{1}(0)$ is the unit ball.

Then, for any $u \in W^{2,2} \cap W_{0}^{1,2}(\Omega)$ we have

$$
\begin{aligned}
\int_{\Omega}(\Delta u)^{2} d x \geq & \frac{n^{2}(n-4)^{2}}{16} \int_{\Omega} \frac{u^{2}}{|x|^{4}} d x \\
& +\frac{n(n-4)}{2} \Lambda(2)\left(\frac{e_{n}}{|\Omega|}\right)^{2 / n} \int_{\Omega} \frac{u^{2}}{|x|^{2}} d x+\Lambda(4)^{2}\left(\frac{e_{n}}{|\Omega|}\right)^{4 / n} \int_{\Omega} u^{2} d x .
\end{aligned}
$$


Combining this result with the Hardy inequality (8) of Brezis-Vazquez, for third order Sobolev spaces we have

Theorem 3. Let $\Omega \subset \mathbb{R}^{n}, n \geq 6$, be a bounded domain. Then for any $u \in$ $W^{3,2} \cap W_{0}^{1,2}(\Omega)$ with $\Delta u=0$ on $\partial \Omega$, i.e. $\Delta u \in W_{0}^{1,2}(\Omega)$, we have:

$$
\begin{aligned}
\int_{\Omega}|\nabla \Delta u|^{2} d x \geq & \frac{(n+2)^{2}(n-2)^{2}(n-6)^{2}}{64} \int_{\Omega} \frac{u^{2}}{|x|^{6}} d x \\
& +\frac{1}{16}\left(3(n-2)^{4}+8(n-2)^{2}+16\right) \Lambda(2)\left(\frac{e_{n}}{|\Omega|}\right)^{2 / n} \int_{\Omega} \frac{u^{2}}{|x|^{4}} d x \\
& +\left(\frac{(n-2)^{2}}{4} \Lambda(4)^{2}+\frac{n(n-4)}{2} \Lambda(2)^{2}\right)\left(\frac{e_{n}}{|\Omega|}\right)^{4 / n} \int_{\Omega} \frac{u^{2}}{|x|^{2}} d x \\
& +\Lambda(2) \Lambda(4)^{2}\left(\frac{e_{n}}{|\Omega|}\right)^{6 / n} \int_{\Omega} u^{2} d x
\end{aligned}
$$

Proof. First, by combining the argument from the proof of Theorem 2 and $\mathrm{AL}$, Theorem 2.7], we reduce the assertion to the case $\Omega=B$ in the radial setting.

An application of the Brezis-Vazquez inequality (8) reduces the order of terms by one. Indeed, we have

$$
\int_{0}^{1} r^{n-1}|\nabla \Delta u|^{2} d r \geq \frac{(n-2)^{2}}{4} \int_{0}^{1} r^{n-3}(\Delta u)^{2} d r+\Lambda(2) \int_{0}^{1} r^{n-1}(\Delta u)^{2} d r .
$$

The second term of this inequality can be estimated with the help of Corollary 1 while the singular term

$$
\int_{0}^{1} r^{n-3}(\Delta u)^{2} d r
$$

can be handled by introducing the transformation

$$
v(r):=r^{(n / 2)-3} u(r), \quad u(r)=r^{3-(n / 2)} v(r) .
$$

We obtain

$$
\begin{aligned}
\int_{0}^{1} r^{n-3}(\Delta u)^{2} d r-\frac{(n+2)^{2}(n-6)^{2}}{16} \int_{0}^{1} r^{n-7} u^{2} d r \\
=\int_{0}^{1} r^{3}\left(\Delta_{4} v+\frac{2}{r} v^{\prime}\right)^{2} d r-\frac{(n+2)(n-6)}{2} \int_{0}^{1} r\left(v^{\prime \prime}+\frac{5}{r} v^{\prime}\right) v d r \\
=\int_{0}^{1} r^{3}\left(\Delta_{4} v\right)^{2} d r+4 \int_{0}^{1} r^{2}\left(\Delta_{4} v\right) v^{\prime} d r \\
\quad+4 \int_{0}^{1} r\left(v^{\prime}\right)^{2} d r+\frac{(n+2)(n-6)}{2} \int_{0}^{1} r\left(-\Delta_{2} v\right) v d r \\
=\quad \int_{0}^{1} r^{3}\left(\Delta_{4} v\right)^{2} d r+2\left[r^{2} v^{\prime}(r)^{2}\right]_{0}^{1}+\frac{1}{2}\left((n-2)^{2}+8\right) \int_{0}^{1} r\left(-\Delta_{2} v\right) v d r \\
\geq \quad \Lambda(4)^{2} \int_{0}^{1} r^{3} v^{2} d r+\frac{1}{2}\left((n-2)^{2}+8\right) \Lambda(2) \int_{0}^{1} r v^{2} d r \\
=\quad \Lambda(4)^{2} \int_{0}^{1} r^{n-3} u^{2} d r+\frac{1}{2}\left((n-2)^{2}+8\right) \Lambda(2) \int_{0}^{1} r^{n-5} u^{2} d r .
\end{aligned}
$$

Note that only the boundary condition $u(1)=v(1)=0$ was used. Collecting terms completes the proof. 
In order to iterate further we quote from [DH, Theorem 12], [Mi, Theorem 3.3] the following

Lemma 2. Let $\Omega \subset \mathbb{R}^{n}$ be a sufficiently smooth bounded domain and $\sigma<n-4$. Then for every $u \in C^{2}(\bar{\Omega})$ with $\left.u\right|_{\partial \Omega}=0$ we have

$$
\int_{\Omega} \frac{(\Delta u)^{2}}{|x|^{\sigma}} d x \geq \frac{(n-4-\sigma)^{2}(n+\sigma)^{2}}{16} \int_{\Omega} \frac{u^{2}}{|x|^{\sigma+4}} d x
$$

We emphasize that for the validity of the above inequality it is enough to assume that $\left.u\right|_{\partial \Omega}=0$.

With the help of this result it is not difficult to prove the existence of remainder terms in Hardy inequalities of arbitrary order. Indeed, we have

Corollary 2. Let $\Omega \subset \mathbb{R}^{n}$ be a sufficiently smooth bounded domain and let $k \in \mathbb{N}$, $2 k \leq n$. Then there exist constants $c_{1}, \ldots, c_{k}$, depending only on $k, n$ and $|\Omega|$, such that for every $u \in W^{k, 2}(\Omega)$ with $\left.\Delta^{j} u\right|_{\partial \Omega}=0$ for $j \in \mathbb{N}_{0}$ and $2 j<k$ we have:

(a) if $k$ is even, $k=2 m, m \in \mathbb{N}$ :

$$
\int_{\Omega}\left(\Delta^{m} u\right)^{2} d x \geq \frac{1}{4^{2 m}}\left(\prod_{j=1}^{2 m}(n+4(m-j))^{2}\right) \int_{\Omega} \frac{u^{2}}{|x|^{4 m}} d x+\sum_{\ell=1}^{2 m} c_{\ell} \int_{\Omega} \frac{u^{2}}{|x|^{4 m-2 \ell}} d x
$$

(b) if $k$ is odd, $k=2 m+1, m \in \mathbb{N}_{0}$ :

$$
\begin{aligned}
\int_{\Omega}\left|\nabla \Delta^{m} u\right|^{2} d x \geq & \frac{1}{4^{2 m+1}}\left(\prod_{j=1}^{2 m+1}(n+4 m+2-4 j)^{2}\right) \int_{\Omega} \frac{u^{2}}{|x|^{4 m+2}} d x \\
& +\sum_{\ell=1}^{2 m+1} c_{\ell} \int_{\Omega} \frac{u^{2}}{|x|^{4 m+2-2 \ell}} d x .
\end{aligned}
$$

The existence of some constants $c_{1}, \ldots, c_{k}$ as above is easily shown. Of particular interest would be their respective optimal values. In this regard, we point out that already the proof of Theorem 3 indicates that iterative methods used to prove this kind of inequalities for functions satisfying the Navier boundary conditions $0=u=\Delta u=\cdots$ on $\partial \Omega$, yield constants which apparently cannot be easily obtained in a closed form.

One may hope that things will improve under the more restrictive Dirichlet boundary conditions, i.e. $u \in W_{0}^{k, 2}(\Omega)$. This will be discussed in the following section.

\section{WeAKly Singular $L^{2}$-REMAINDER terms in RELLICh-TYPE INEQUaLities IN HIGHER-ORDER SOBOLEV SPACES UNDER DIRICHLET BOUNDARY CONDITIONS}

Our first goal in this section will be to adapt Corollary 1 in the case $p=2$, for functions satisfying Dirichlet boundary conditions, i.e. for functions belonging to the space $W_{0}^{2,2}(\Omega)$ instead of $W^{2,2} \cap W_{0}^{1,2}(\Omega)$. At least when $\Omega$ is a ball or "close" to a ball, the estimation constants will be considerably larger. On the other hand, the symmetrization argument used in the proof of Theorem 2 fails: the function $w$ used in that proof does not belong to the space $W_{0}^{2,2}(B)$ considered here. We overcome this difficulty by a relatively involved argument based on the positivity 
properties of Green's functions, which seems to work only in the Hilbert space case $p=2$ and if $\Omega$ is replaced with the circumscribed ball.

Since the technical difficulties for obtaining explicit "large" constants in the inequalities that follow increase quickly with the order $k$ of the space $W_{0}^{k, 2}(\Omega)$, we study the improved Hardy inequality only in $W_{0}^{2,2}(\Omega)$. For the general case we formulate a conjecture on which behaviour of the remainder terms we expect when the order of spaces becomes arbitrarily large.

In this section the eigenvalues of biharmonic and more general polyharmonic operators will play an important role.

Let $B \subset \mathbb{R}^{n}$ denote the unit ball and let

$$
\Lambda\left((-\Delta)^{k}, n\right)=\left\{\begin{array}{cl}
\inf _{W_{0}^{k, 2}(B) \backslash\{0\}} \frac{\int_{B}\left(\Delta^{m} u\right)^{2} d x}{\int_{B} u^{2} d x}, & k=2 m, m \in \mathbb{N} ; \\
\inf _{W_{0}^{k, 2}(B) \backslash\{0\}} \frac{\int_{B}\left|\nabla \Delta^{m} u\right|^{2} d x}{\int_{B} u^{2} d x}, & k=2 m+1, m \in \mathbb{N}_{0} .
\end{array}\right.
$$

The notation $\Lambda(n)$ of Section 3 is a special case of the notation introduced here; indeed we have $\Lambda(n)=\Lambda((-\Delta), n)$.

Remark. Taking Proposition 1 into account we immediately see that $\Lambda\left((-\Delta)^{2}, n\right) \geq$ $\Lambda(-\Delta, n)^{2}$. With the help of qualitative properties of first eigenfunctions (in particular under Navier boundary conditions), it can be shown that also the strict inequality holds. Moreover, the ratio of these eigenvalues is rather large; by elementary calculations one finds e.g. $\Lambda\left((-\Delta)^{2}, 1\right)=31.285243 \ldots$ while $\Lambda((-\Delta), 1)^{2}=$ $\pi^{4} / 16=6.088068 \ldots$ For $n=4$, the case which is needed below, a rough estimate according to [PS p. 57] gives $\Lambda\left((-\Delta)^{2}, 4\right) \geq j_{1}^{2} j_{2}^{2}=387.23 \ldots$ while $\Lambda((-\Delta), 4)^{2}=j_{1}^{4}=215.56 \ldots$

Theorem 4. Let $n \geq 4$ and let $\Omega \subset \mathbb{R}^{n}$ be such that $\Omega \subset B_{R}(0)$. Then for every $u \in W_{0}^{2,2}(\Omega)$ we have

$$
\begin{aligned}
\int_{\Omega}(\Delta u)^{2} d x \geq & \frac{n^{2}(n-4)^{2}}{16} \int_{\Omega} \frac{u^{2}}{|x|^{4}} d x \\
& +\frac{n(n-4)}{2} \Lambda(-\Delta, 2) R^{-2} \int_{\Omega} \frac{u^{2}}{|x|^{2}} d x \\
& +\Lambda\left((-\Delta)^{2}, 4\right) R^{-4} \int_{\Omega} u^{2} d x .
\end{aligned}
$$

Proof. By trivial extension, we have $W_{0}^{2,2}(\Omega) \hookrightarrow W_{0}^{2,2}\left(B_{R}(0)\right)$. Further, a scaling argument shows that we may assume $R=1\left(B_{R}=B\right)$.

If in addition, $u$ is radially symmetric, we proceed as in Step 2 of the proof of Theorem 2 with $p=2$. In its last conclusion we may instead exploit that $u$ satisfies homogeneous Dirichlet boundary conditions and replace $\left.\Lambda_{\vartheta}\right|_{\vartheta=4}=\Lambda(4)^{2}=$ $\Lambda(-\Delta, 4)^{2}$ with $\Lambda\left((-\Delta)^{2}, 4\right)$, thereby proving (21) for radial $u$.

It remains to extend (21) to arbitrary functions $u \in W_{0}^{2,2}(B)$. To this end, consider for $\ell \in \mathbb{N}$ the sequence of relaxed minimum problems:

$$
\mu_{\ell}:=\inf _{W_{0}^{2,2}(B) \backslash\{0\}} \frac{F_{\ell}(v)}{\int_{B} v^{2} d x},
$$


where

$$
\begin{aligned}
F_{\ell}(v)= & \int_{B}(\Delta v)^{2} d x-\frac{n^{2}(n-4)^{2}}{16}\left(1-\frac{1}{\ell}\right) \int_{B} \frac{v^{2}}{|x|^{4}} d x \\
& -\frac{n(n-4)}{2} \Lambda(-\Delta, 2)\left(1-\frac{1}{\ell}\right) \int_{B} \frac{v^{2}}{|x|^{2}} d x .
\end{aligned}
$$

Since we may already use Corollary 1, we have $F_{\ell}(v) \geq \frac{1}{\ell} \int_{B}(\Delta v)^{2} d x$ for every $v \in W_{0}^{2,2}(B)$. This means that for every minimizing sequence $v_{k} \in W_{0}^{2,2}(B)$ with $\int_{B} v_{k}^{2} d x=1$ and $\lim _{k \rightarrow \infty} F_{\ell}\left(v_{k}\right)=\mu_{\ell}$, the sequence $\left(\left\|u_{k}\right\|_{W_{0}^{2,2}(B)}\right)_{k \in \mathbb{N}}$ is bounded. After selecting a subsequence we may assume that $u_{k} \rightarrow u \in W_{0}^{2,2}(B)$ and $u_{k} \rightarrow u$ strongly in $L^{2}(B)$. The bilinear form

$$
\begin{aligned}
\Phi_{\ell}(v, w):= & \int_{B} \Delta v \cdot \Delta w d x-\frac{n^{2}(n-4)^{2}}{16}\left(1-\frac{1}{\ell}\right) \int_{B} \frac{v \cdot w}{|x|^{4}} d x \\
& -\frac{n(n-4)}{2} \Lambda(-\Delta, 2)\left(1-\frac{1}{\ell}\right) \int_{B} \frac{v \cdot w}{|x|^{2}} d x
\end{aligned}
$$

associated with $F_{\ell}(v)$ defines a scalar product on $W_{0}^{2,2}(B)$, which, by

$$
\frac{1}{\ell} \int_{B}(\Delta v)^{2} d x \leq \Phi_{\ell}(v, v) \leq \int_{B}(\Delta v)^{2} d x=\|v\|_{W_{0}^{2,2}(B)},
$$

defines an equivalent norm. If we consider, just for the following argument, $W_{0}^{2,2}(B)$ with $\Phi_{\ell}(.,$.$) as scalar product, then, the lower semicontinuity of the corresponding$ norm in the weak topology gives

$$
\mu_{\ell}=\liminf _{k \rightarrow \infty} F_{\ell}\left(v_{k}\right) \geq F_{\ell}(v)
$$

Further, we have

$$
1=\lim _{k \rightarrow \infty} \int_{B} v_{k}^{2} d x=\int_{B} v^{2} d x .
$$

Hence $F_{\ell}(v)=\mu_{\ell}$, and $v \in W_{0}^{2,2}(B)$ is an optimal (nontrivial) function for $\mu_{\ell}$. Consequently, $v$ is a weak solution of the Euler-Lagrange equation

$$
\left\{\begin{array}{rlrl}
\Delta^{2} v= & \left(1-\frac{1}{\ell}\right) \frac{n^{2}(n-4)^{2}}{16} \frac{v}{|x|^{4}} & \\
& +\left(1-\frac{1}{\ell}\right) \frac{n(n-4)}{2} \Lambda(-\Delta, 2) \frac{v}{|x|^{2}}+\mu_{\ell} v & & \text { in } B \\
v=\nabla v & =0 & & \text { on } \partial B .
\end{array}\right.
$$

Next we show that $v$ is of fixed sign. Assume by contradiction that there exist subsets $B^{+}, B^{-} \subset B$, both of positive measure, such that $v>0$ on $B^{+}$and $v<0$ on $B^{-}$. We may now apply a decomposition method explained in detail in GG. Section 3]; see also [Mo]. Let

$$
\mathcal{K}=\left\{u \in W_{0}^{2,2}(B): u \geq 0\right\}
$$

be the closed convex cone of nonnegative functions and

$$
\mathcal{K}^{*}=\left\{u^{*} \in W_{0}^{2,2}(B):\left(u^{*}, u\right) \leq 0 \text { for all } u \in \mathcal{K}\right\}
$$

the dual cone. Here $\left(u^{*}, u\right)=\int_{B}\left(\Delta u^{*}\right)(\Delta u) d x$ is the standard scalar product in $W_{0}^{2,2}(B)$ and $\mathcal{K}^{*}$ is the cone of all weak subsolutions of the biharmonic equation 
in $B$ under Dirichlet boundary conditions. By a comparison result of Boggio $\mathrm{BO}$ p. 126] the elements of $\mathcal{K}^{*}$ are nonpositive. The strict positivity of the biharmonic Green function in $B$ even shows that $u^{*} \in \mathcal{K}^{*} \backslash\{0\} \Rightarrow u^{*}<0$; cf. AGGM, Lemma 1]. According to $[\mathrm{Mo}$, we decompose

$$
v=v_{1}+v_{2}, \quad v_{1} \in \mathcal{K}, \quad v_{2} \in \mathcal{K}^{*}, \quad v_{1} \perp v_{2} .
$$

Since $0 \not \equiv v_{1} \geq 0, v_{2}<0$, replacing $v=v_{1}+v_{2}$ with $v_{1}-v_{2} \geq 0$ yields

$$
F_{\ell}\left(v_{1}+v_{2}\right)>F_{\ell}\left(v_{1}-v_{2}\right), \quad \int_{B}\left(v_{1}+v_{2}\right)^{2} d x<\int_{B}\left(v_{1}-v_{2}\right)^{2} d x,
$$

contradicting the minimality of $F_{\ell}(v) / \int_{B} v^{2} d x$.

Hence we may assume that $0 \not \equiv v \geq 0$. Considering polar coordinates $x=r \xi$, $r \in[0,1],|\xi|=1$ and integrating the Euler-Lagrange equation (22) over $\{|\xi|=1\}$ shows that

$$
w(r):=\frac{1}{n e_{n}} \int_{|\xi|=1} v(r \xi) d \omega(\xi) \in W_{0}^{2,2}(B)
$$

is a radial weak solution of (22). By virtue of $0 \not \equiv v \geq 0$, we also have $0 \not \equiv w \geq 0$. Since we may already use the Hardy inequality (21) for the radial function $w$, we conclude that

$$
\begin{aligned}
\mu_{\ell} & =\frac{F_{\ell}(w)}{\int_{B} w^{2} d x} \\
& >\frac{\int_{B}(\Delta w)^{2} d x-\frac{n^{2}(n-4)^{2}}{16} \int_{B} \frac{w^{2}}{|x|^{4}} d x-\frac{n(n-4)}{2} \Lambda(-\Delta, 2) \int_{B} \frac{w^{2}}{|x|^{2}} d x}{\int_{B} w^{2} d x} \\
& \geq \Lambda\left((-\Delta)^{2}, 4\right) .
\end{aligned}
$$

To sum up, we have shown that for any $\ell \in \mathbb{N}$ and for every $u \in W_{0}^{2,2}(B)$ we have

$$
\begin{aligned}
\int_{B}(\Delta u)^{2} d x \geq & \left(1-\frac{1}{\ell}\right) \frac{n^{2}(n-4)^{2}}{16} \int_{B} \frac{u^{2}}{|x|^{4}} d x \\
& +\left(1-\frac{1}{\ell}\right) \frac{n(n-4)}{2} \Lambda(-\Delta, 2) \int_{B} \frac{u^{2}}{|x|^{2}} d x \\
& +\Lambda\left((-\Delta)^{2}, 4\right) \int_{B} u^{2} d x .
\end{aligned}
$$

Sending $\ell \rightarrow \infty$ in the above inequality, the claim follows.

We conclude this section with some remarks concerning general higher-order Sobolev spaces.

We recall that $W_{0}^{k, 2}(\Omega)$ is equipped with the norm

$$
\|u\|_{W_{0}^{k, 2}(\Omega)}^{2}=\left\{\begin{array}{l}
\int_{\Omega}\left(\Delta^{m} u\right)^{2} d x, \quad \text { if } k=2 m, m \in \mathbb{N} ; \\
\int_{\Omega}\left|\nabla \Delta^{m} u\right|^{2} d x, \quad \text { if } k=2 m+1, m \in \mathbb{N}_{0} .
\end{array}\right.
$$


We expect the following result:

Conjecture 1. Let $\Omega \subset \mathbb{R}^{n}$ be such that $\Omega \subset B_{R}(0)$. Let $k \in \mathbb{N}, n \geq 2 k$. Then for all $u \in W_{0}^{k, 2}(\Omega)$ we have:

$$
\begin{aligned}
\|u\|_{W_{0}^{k, 2}(\Omega)}^{2} \geq & \sum_{j=0}^{k} \frac{1}{4^{j}}\left(\begin{array}{l}
k \\
j
\end{array}\right)\left\{\prod_{\ell=1}^{j}((n+2 k-4 \ell)(n-2 k-4+4 \ell))\right\} \\
& \cdot \Lambda\left((-\Delta)^{k-j}, 2 k-2 j\right) R^{2 j-2 k} \int_{\Omega} \frac{u^{2}}{|x|^{2 j}} d x
\end{aligned}
$$

with the convention that $\Lambda\left((-\Delta)^{0}, 0\right)=1$.

In the radial setting, the terms

originate from

$$
\Lambda\left((-\Delta)^{k-j}, 2 k-2 j\right) \int_{B} \frac{u^{2}}{|x|^{2 j}} d x
$$

$$
n e_{n} \int_{0}^{1} r^{2 k-2 j-1}\left(\Delta_{2 k-2 j}^{(k-j) / 2} v\right)^{2} d r
$$

if $k-j$ is even, and from

$$
n e_{n} \int_{0}^{1} r^{2 k-2 j-1}\left(\left(\Delta_{2 k-2 j}^{(k-j-1) / 2} v\right)^{\prime}\right)^{2} d r
$$

if $k-j$ is odd. Here $\Delta_{2 k-2 j}$ is the radial Laplacian in dimension $2 k-2 j$. The radial functions $u$ and $v$ are related by means of the transformation

$$
v(r)=r^{(n / 2)-k} u(r), \quad u(r)=r^{k-(n / 2)} v(r) .
$$

In Conjecture 1 as well as in the proof of Theorem 4 we simply used eigenvalue estimates for these terms. One may also wish to use Hardy-like inequalities in order to have largest possible constants in front of the most singular remainder terms. Since we are assuming that we are dealing with functions satisfying homogeneous Dirichlet boundary conditions, we can apply the following lemma.

Lemma 3. (a) Let $k \geq 2, j \geq 1, v \in C^{2}([0,1])$ with $v^{\prime}(1)=0$. Then:

$$
\int_{0}^{1} r^{k}\left(\Delta_{j} v\right)^{2} d r \geq \frac{(k+1-2 j)^{2}}{4} \int_{0}^{1} r^{k-2}\left(v^{\prime}\right)^{2} d r
$$

(b) Let $k \geq 2, v \in C^{1}([0,1])$ with $v(1)=0$. Then:

$$
\int_{0}^{1} r^{k}\left(v^{\prime}\right)^{2} d r \geq \frac{(k-1)^{2}}{4} \int_{0}^{1} r^{k-2} v^{2} d r .
$$

To illustrate how the application of this elementary and well-known lemma shifts less singular remainder terms to more singular ones, we modify the proof of Theorem 4 and obtain:

Corollary 3. Let $n \geq 4$ and let $\Omega \subset \mathbb{R}^{n}$ be such that $\Omega \subset B_{R}(0)$. Then for every $u \in W_{0}^{2,2}(\Omega)$ we have

$$
\begin{aligned}
\int_{\Omega}(\Delta u)^{2} d x \geq & \frac{n^{2}(n-4)^{2}}{16} \int_{\Omega} \frac{u^{2}}{|x|^{4}} d x \\
& +\frac{1}{2}(n(n-4)+8) \Lambda(-\Delta, 2) R^{-2} \int_{\Omega} \frac{u^{2}}{|x|^{2}} d x .
\end{aligned}
$$




\section{Appendix A. Proofs of some technical inequalities}

First we present a proof of Lemma 1 from Section 2 .

Proof of Lemma[1. By means of integration by parts or Taylor's formula we find

$$
(\xi-\eta)^{p}+p \xi^{p-1} \eta-\xi^{p}=p(p-1) \eta^{2} \int_{0}^{1}(1-t)(\xi-t \eta)^{p-2} d t
$$

Assume that $p \geq 2$. Since $\xi-\eta \geq 0$, we have $\xi-t \eta \geq(1-t) \xi$ and the right hand side in (24) becomes

$p(p-1) \eta^{2} \int_{0}^{1}(1-t)(\xi-t \eta)^{p-2} d t \geq p(p-1) \eta^{2} \xi^{p-2} \int_{0}^{1}(1-t)^{p-1} d t=(p-1) \eta^{2} \xi^{p-2}$.

To get the second estimate from below in the case $p \geq 2$, we first consider nonpositive $\eta \leq 0$. Then $\xi-t \eta \geq t|\eta|$ and the right hand side in (24) becomes

$$
p(p-1) \eta^{2} \int_{0}^{1}(1-t)(\xi-t \eta)^{p-2} d t \geq p(p-1)|\eta|^{p} \int_{0}^{1}(1-t) t^{p-2} d t=|\eta|^{p} .
$$

For $\eta \geq 0$ we get $\xi-t \eta \geq(1-t)|\eta|$ and thus

$p(p-1) \eta^{2} \int_{0}^{1}(1-t)(\xi-t \eta)^{p-2} d t \geq p(p-1)|\eta|^{p} \int_{0}^{1}(1-t)^{p-1}=(p-1)|\eta|^{p} \geq|\eta|^{p}$.

Now let $1 \leq p<2$. This implies $(\xi-t \eta)^{p-2} \geq(\xi+|\eta|)^{p-2}$, and consequently

$$
\begin{aligned}
p(p-1) \eta^{2} \int_{0}^{1}(1-t)(\xi-t \eta)^{p-2} d t & \geq \frac{p(p-1) \eta^{2}}{(\xi+|\eta|)^{2-p}} \int_{0}^{1}(1-t) d t \\
& =\frac{p(p-1)}{2} \frac{\eta^{2}}{(\xi+|\eta|)^{2-p}} .
\end{aligned}
$$

This completes the proof.

We now refer to the estimate on $\Gamma$ and $\Lambda_{\vartheta}$ mentioned in the first remark of Section 3 Clearly we have $\Lambda_{\vartheta} \geq 0$. We shall show that even $\Lambda_{\vartheta}>0$ and $\Lambda_{\vartheta} \rightarrow \infty$ as $n \rightarrow \infty$ whenever $p>2$.

Lemma 4. Let $X=\left\{v \in C^{2}([0,1]): v^{\prime}(0)=v(1)=0, v \not \equiv 0\right\}$. Assume that $p>2$; then we have

$$
\begin{gathered}
\Lambda_{\vartheta} \stackrel{\text { def }}{=} \inf _{X} \frac{\int_{0}^{1} r^{3}|v(r)|^{p-2}\left(\Delta_{\vartheta} v(r)\right)^{2} d r}{\int_{0}^{1} r^{2 p-1}|v(r)|^{p} d r} \geq 4 \frac{n^{2}(p-2)^{2}}{p^{2}}>0, \\
\lambda_{\vartheta} \stackrel{\text { def }}{=} \inf _{X} \frac{\int_{0}^{1} r^{2 p-1}\left|\Delta_{\vartheta} v(r)\right|^{p} d r}{\int_{0}^{1} r^{2 p-1}|v(r)|^{p} d r} \geq 2^{p} \frac{n^{p}(p-2)^{p}}{p^{p}}>0 .
\end{gathered}
$$

Proof. Let $v \in X$. A first integration by parts shows that

$$
\int_{0}^{1} r^{2 p-1}|v(r)|^{p} d r=-\frac{1}{2} \int_{0}^{1} r^{2 p}|v(r)|^{p-2} v(r) v^{\prime}(r) d r .
$$

By means of the Cauchy-Schwarz inequality we obtain

$$
\int_{0}^{1} r^{2 p-1}|v(r)|^{p} d r \leq \frac{1}{4} \int_{0}^{1} r^{2 p+1}|v(r)|^{p-2}\left(v^{\prime}(r)\right)^{2} d r .
$$


Recalling that from Section 3 we have $\vartheta=4+\frac{n(p-2)}{p}$, a further integration by parts gives

$$
\begin{aligned}
& (n(p-2)-2 p(p-1)) \int_{0}^{1} r^{2 p}|v(r)|^{p-2} v(r) v^{\prime}(r) d r \\
& =p(p-1) \int_{0}^{1} r^{2 p+1}|v(r)|^{p-2}\left(v^{\prime}(r)\right)^{2} d r+p \int_{0}^{1} r^{2 p+1}|v(r)|^{p-2} v(r) \Delta_{\vartheta} v(r) d r .
\end{aligned}
$$

Combining (27), (28) and (26) yields

$$
\begin{aligned}
4 \int_{0}^{1} r^{2 p-1}|v(r)|^{p} d r \leq \int_{0}^{1} r^{2 p+1}|v(r)|^{p-2}\left|v^{\prime}(r)\right|^{2} d r \\
=\frac{n(p-2)-2 p(p-1)}{p(p-1)} \int_{0}^{1} r^{2 p}|v(r)|^{p-2} v(r) v^{\prime}(r) d r \\
\quad-\frac{1}{p-1} \int_{0}^{1} r^{2 p+1}|v(r)|^{p-2} v(r) \Delta_{\vartheta} v(r) d r \\
=-2\left(\frac{n(p-2)}{p(p-1)}-2\right) \int_{0}^{1} r^{2 p-1}|v(r)|^{p} d r \\
\quad-\frac{1}{p-1} \int_{0}^{1} r^{2 p+1}|v(r)|^{p-2} v(r) \Delta_{\vartheta} v(r) d r
\end{aligned}
$$

from which we infer at once that (recall $r \leq 1$ )

$$
\begin{aligned}
& 2 \frac{n(p-2)}{p} \int_{0}^{1} r^{2 p-1}|v(r)|^{p} d r \leq-\int_{0}^{1} r^{2 p+1}|v(r)|^{p-2} v(r) \Delta_{\vartheta} v(r) d r \\
& \quad \leq \int_{0}^{1}\left(r^{p-1 / 2}|v(r)|^{p / 2}\right)\left(r^{3 / 2}|v(r)|^{(p-2) / 2}\left|\Delta_{\vartheta} v(r)\right|\right) r^{p} d r \\
& \quad \leq\left(\int_{0}^{1} r^{2 p-1}|v(r)|^{p} d r\right)^{1 / 2}\left(\int_{0}^{1} r^{3}|v(r)|^{p-2}\left(\Delta_{\vartheta} v(r)\right)^{2} d r\right)^{1 / 2},
\end{aligned}
$$

and the first claim follows. In order to obtain the second statement, we only need to modify this last estimate as follows:

$$
\begin{aligned}
& 2 \frac{n(p-2)}{p} \int_{0}^{1} r^{2 p-1}|v(r)|^{p} d r \leq-\int_{0}^{1} r^{2 p+1}|v(r)|^{p-2} v(r) \Delta_{\vartheta} v(r) d r \\
& \leq \int_{0}^{1}\left(r^{(2 p-1)(p-1) / p}|v(r)|^{p-1}\right)\left(r^{(2 p-1) / p}\left|\Delta_{\vartheta} v(r)\right|\right) r^{2} d r \\
& \quad \leq\left(\int_{0}^{1} r^{2 p-1}|v(r)|^{p} d r\right)^{(p-1) / p}\left(\int_{0}^{1} r^{2 p-1}\left|\Delta_{\vartheta} v(r)\right|^{p} d r\right)^{1 / p} .
\end{aligned}
$$

This completes the proof of Lemma 5 .

\section{ACKNOWLEDGEMENT}

We are grateful to the referees and to Professor William Beckner for useful comments and suggestions on how to improve the exposition of the present work. 


\section{REFERENCES}

[ACR] Adimurthi, N. Chaudhuri, M. Ramaswamy, An improved Hardy-Sobolev inequality and its application, Proc. Amer. Math. Soc. 130 (2002), 489-505. MR 2002j:35232

[AE] Adimurthi, M.J. Esteban, An improved Hardy-Sobolev inequality in $W^{1, p}$ and its application to Schrödinger operator, Nonlinear Differ. Eq. Appl. NoDEA, to appear.

[AL] F. Almgren, E. Lieb, Symmetric decreasing rearrangement is sometimes continuous, $J$. Amer. Math. Soc. 2 (1989), 683-773. MR 90f:49038

[AGGM] G. Arioli, F. Gazzola, H.-Ch. Grunau, E. Mitidieri, A semilinear fourth order elliptic problem with exponential nonlinearity, preprint.

[BFT] G. Barbatis, S. Filippas, A. Tertikas, A unified approach to improved $L^{p}$ Hardy inequalities with best constants, Trans. Amer. Math. Soc., this issue.

[Bo] T. Boggio, Sulle funzioni di Green d'ordine m, Rend. Circ. Mat. Palermo 20 (1905), 97-135.

[BL] H. Brezis, E. Lieb, Sobolev inequalities with remainder terms, J. Funct. Anal. 62 (1985), 73-86. MR 86i:46033

[BM] H. Brezis, M. Marcus, Hardy's inequalities revisited, Ann. Sc. Norm. Sup. Pisa 25 (1997), 217-237. MR 99m:46075

[BMS] H. Brezis, M. Marcus, I. Shafrir, Extremal functions for Hardy's inequality with weight, J. Funct. Anal. 171 (2000), 177-191. MR 2001g:46068

[BV] H. Brezis, J. L. Vazquez, Blow-up solutions of some nonlinear elliptic problems, Rev. Mat. Univ. Complutense Madrid 10 (1997), 443-469. MR 99a:35081

[DA] L. D'Ambrosio, Some Hardy inequalities on the Heisenberg group, Differential Equations, in press.

[DH] E. B. Davies, A. M. Hinz, Explicit constants for Rellich inequalities in $L_{p}(\Omega)$, Math. Z. 227 (1998), 511-523. MR 99e:58169

[E] H. Egnell, Existence and nonexistence results for $m$-Laplace equations involving critical Sobolev exponents, Arch. Ration. Mech. Anal. 104 (1988), 57-77. MR 90e:35069

[FT] S. Filippas, A. Tertikas, Optimizing improved Hardy inequalities, J. Funct. Anal. 192 (2002), 186-233. MR 2003f:46045

[GG] F. Gazzola, H.-Ch. Grunau, Critical dimensions and higher order Sobolev inequalities with remainder terms, Nonlin. Diff. Eq. Appl. NoDEA 8 (2001), 35-44. MR 2002d:35024

[H] G. H. Hardy, Notes on some points in the integral calculus, Messenger Math. 48 (1919), 107-112.

[HLP] G. H. Hardy, J. E. Littlewood, G. Pólya, Inequalities, Cambridge: University Press, 1934.

[L] P. Lindqvist, On the equation $\operatorname{div}\left(|\nabla u|^{p-2} \nabla u\right)+\lambda|u|^{p-2} u=0$, Proc. Amer. Math. Soc. 109 (1990), 157-164. MR 90h:35088

[MS] M. Marcus, I. Shafrir, An eigenvalue problem related to Hardy's $L^{p}$ inequality, Ann. Scuola Norm. Sup. Pisa Ser. IV 29 (2000), 581-604. MR 2002c: 49082

[Ma] V. G. Maz'ya, Sobolev spaces, Transl. from the Russian by T. O. Shaposhnikova, Berlin etc.: Springer-Verlag, 1985. MR 87g:46056

[Mi] E. Mitidieri, A simple approach to Hardy's inequalities, Math. Notes 67 (2000), 479-486, translation from Mat. Zametki 67 (2000), 563-572. MR 2001f:26022

[Mo] J. J. Moreau, Décomposition orthogonale d'un espace hilbertien selon deux cônes mutuellement polaires, C. R. Acad. Sci. Paris 255 (1962), 238-240. MR 25:3346

[PS] P. Pucci, J. Serrin, Critical exponents and critical dimensions for polyharmonic operators, J. Math. Pures Appl. 69 (1990), 55-83. MR 91i:35065

[R] F. Rellich, Halbbeschränkte Differentialoperatoren höherer Ordnung, in: J. C. H. Gerretsen et al. (eds.), Proceedings of the International Congress of Mathematicians Amsterdam 1954, Vol. III, pp. 243-250, Groningen: Nordhoff, 1956. MR 19:550c

[Ta] G. Talenti, Elliptic equations and rearrangements, Ann. Scuola Norm. Sup. Pisa Ser. IV 3 (1976), 697-718. MR 58:29170

[To] P. Tolksdorf, Regularity for a more general class of quasilinear equations, J. Differ. Equations 51 (1984), 126-150. MR 85g:35047 
[VZ] J. L. Vazquez, E. Zuazua, The Hardy constant and the asymptotic behaviour of the heat equation with an inverse-square potential, J. Funct. Anal. 173 (2000), 103-153. MR 2001j:35122

[V] R. van der Vorst, Best constant for the embedding of the space $H^{2} \cap H_{0}^{1}(\Omega)$ into $L^{2 N /(N-4)}(\Omega)$, Diff. Int. Eq. 6 (1993), 259-276. MR 94b:46053

Dipartimento di Matematica, Politecnico di Milano, Piazza leonardo da Vinci, 32, I-20133 Milano, Italy

E-mail address: gazzola@mate.polimi.it

Fakultät für Mathematik, Otto-von-Guericke-Universität, Postfach 4120, D-39016 Magdeburg, Germany

E-mail address: Hans-Christoph.Grunau@mathematik.uni-magdeburg.de

Dipartimento di Scienze Matematiche, Via A. Valerio 12/1, Università degli Studi di Trieste, I-34100 Trieste, Italy

E-mail address: mitidier@univ.trieste.it 